\title{
LncRNA NR024118 is downregulated in sepsis and inhibits LPS-induced apoptosis of cardiomyocytes
}

\author{
GANG QIN*, LIUQING WEI* , FUFU JIANG, JIAYAN LI, BO ZHANG, DONGXU PAN and XINGLIN LU \\ ICU Department, Guangxi Zhuang Autonomous National Hospital, Nanning, \\ Guangxi Zhuang Autonomous Region 530001, P.R. China
}

Received July 23, 2020; Accepted January 13, 2021

DOI: $10.3892 / \mathrm{mmr} .2021 .12073$

\begin{abstract}
It has been reported that lncRNA-NR024118 can suppress lipopolysaccharide (LPS)-induced inflammatory responses, which promote sepsis. The present study aimed to investigate the involvement of NR024118 in sepsis. Research subjects included 82 patients with sepsis without myocardial dysfunction (MD), 35 patients with sepsis with MD and 82 healthy controls. The expression levels of NR024118 in plasma collected from these participants and LPS-induced AC16 cells were measured by reverse transcription-quantitative PCR. The expression levels of IL-16 in these plasma samples and LPS-induced AC16 cells were measured by ELISA. The correlation between the expression levels of NR024118 and IL- 6 across plasma samples were analyzed by Pearson's correlation coefficient. The action potential duration (APD) was measured at 50 and $90 \%$ repolarization. Cell apoptosis was determined by cell apoptosis assay. The expression levels of p-transcription factor p65 were detected by western blot analysis. NF- $\kappa \mathrm{B}$ activity were analyzed by luciferase reporter assay. It was found that NR024118 was downregulated and IL-6 was upregulated in the plasma of patients with sepsis. Among patients with sepsis, the individuals with MD exhibited even lower expression levels of NR024118 and higher expression levels of IL-6. Among patients with sepsis with MD, the expression levels of NR024118 and IL-6 were inversely correlated. LPS could induce MD to construct the sepsis models based
\end{abstract}

Correspondence to: Dr Fufu Jiang or Dr Xinglin Lu, ICU Department, Guangxi Zhuang Autonomous National Hospital, 232 Mingxiu East Road, Nanning, Guangxi Zhuang Autonomous Region 530001, P.R. China

E-mail: tx6142@163.com

E-mail: osnwsekagh9@163.com

${ }^{*}$ Contributed equally

Abbreviations: MD, myocardial dysfunction; ncRNAs, non-coding RNAs; lncRNAs, long (>200 nt) ncRNAs

Key words: NR024118, sepsis, myocardial dysfunction, IL-6, apoptosis, cardiomyocytes on the increased expression levels of TNF- $\alpha$, IL-1 $\beta$, IL-6 and shortened APD by LPS-mediated induction. Overexpression of NR024118 significantly reduced the secretion of IL-6 and apoptosis of cardiomyocytes under LPS treatment. Functional studies demonstrated that NR024118 had negative regulation on $\mathrm{p} 65$ phosphorylation and NF- $\kappa \mathrm{B}$ activation. NR024118 was suppressed in sepsis and inhibited LPS-induced apoptosis of cardiomyocytes.

\section{Introduction}

Sepsis is a type of clinical disorder characterized by dysregulated immune and systemic inflammatory response to infections (1) and is induced by pathogens such as lipopolysaccharide (LPS)-releasing gram-negative bacteria. Sepsis can cause severe injuries in multiple organs, leading to a mortality rate ranging $15-25 \%$ (2). Without timely diagnosis and treatment, sepsis can cause irreversible septic shock, tissue damage and failure of multiple organs. Therefore, early diagnosis remains the key for the survival of patients with sepsis (3). Myocardial dysfunction (MD) is a common complication of sepsis $(4,5)$. MD is mainly caused by global myocardial ischemia in sepsis (5). The early onset of sepsis-induced MD lacks obvious and classic clinical symptoms and the early detection is poor, leading to inferior treatment outcomes (6).

Studies on the pathogenesis of sepsis have identified many molecular factors involved in the occurrence and progression of sepsis $(7,8)$. The development of sepsis requires the involvement of non-coding RNAs (ncRNAs), such as long (>200 nt) ncRNAs (lncRNAs) (9). Some lncRNAs have been demonstrated to be critical factors in sepsis and regulation of their expression is considered as a potential therapeutic target for sepsis $(10,11)$. However, the functions of most lncRNAs in sepsis remain to be elucidated. LncRNA NR024118 has been characterized as an inhibitor of LPS-induced inflammatory injury (12), a key player in sepsis (13). Preliminary RNA-seq data in the present study revealed an inverse correlation between NR024118 and IL-6, which promotes inflammatory responses in patients with sepsis (14). Therefore, it was hypothesized that NR024118 may also participate in sepsis. The present study was therefore performed to investigate the interaction between NR024118 and IL-6 in sepsis. 


\section{Materials and methods}

Patients with sepsis and healthy controls. The present study was approved by the Ethics Committee of Guangxi Zhuang Autonomous National Hospital (China; approval no. 35765323). Research subjects included 82 patients with sepsis but without MD (Sepsis group; 50 males and 32 females, age range 37-67 years old, mean age 52.1 \pm 6.0 years old), 35 patients with sepsis and MD (MD group; 23 males and 12 females, age range $37-68$ years old, mean age 51.8 \pm 6.4 years old) and 82 healthy controls (Control group; 50 males and 32 females, age range $37-67$ years old, mean age $52.3 \pm 6.3$ years old). In all patients with sepsis included in the present study sepsis was caused by bacterial infection. Patients with sepsis were diagnosed by blood test. MD was diagnosed by echocardiogram (ejection fraction below 45\%), blood tests and symptoms, such as shoulder or arm pain, shortness of breath and fatigue. The present study excluded patients with MD caused by factors other than sepsis. All participants were enrolled at aforementioned hospital between March 2017 and March 2019. Patients were all newly diagnosed cases and recurrent cases were excluded. Exclusion criteria included: i) Patients afflicted with other diseases, such as cancer and metabolic diseases; and ii) patients who were treated by any therapies prior to admission. All patients signed informed consent before this study. Baseline clinical data of three groups of participants are listed in Table I.

Plasma preparation. On the day of admission all participants were subjected to blood $(5 \mathrm{ml})$ extraction under fasting conditions. All blood samples were transferred to BD Vacutainer ${ }^{\circledR}$ PPT $^{\mathrm{TM}}$ plasma preparation tubes (BD Diagnostics; Becton, Dickinson and Company) to be centrifuged at $1,200 \mathrm{x} \mathrm{g}$ at room temperature for $12 \mathrm{~h}$ to prepare plasma samples. Plasma samples were stored at $-80^{\circ} \mathrm{C}$ before use.

Cardiomyocytes and cell culture. The human cardiomyocyte cell line AC16 was purchased from Sigma-Aldrich (Merck $\mathrm{KGaA})$. Cell culture medium was composed of $10 \%$ FBS and 90\% cardiomyocyte growth medium (ScienCell Research Laboratories, Inc.). Cells were cultivated in a $5 \% \mathrm{CO}_{2}$ incubator at $37^{\circ} \mathrm{C}$ to reach the confluence of $\sim 80 \%$. AC16 cells were treated with LPS at different concentrations $(0,150,300$ and $500 \mathrm{ng} / \mathrm{ml}$ ) for $24 \mathrm{~h}$ to induce sepsis models.

Vector construction and transient transfections. PcDNA3.1 vector was used as the backbone to establish the expression vector of NR024118 (NCBI ID: NR_024118.1). The vector construction service was provided by Invitrogen (Thermo Fisher Scientific, Inc.). The short interfering RNAs (siRNAs) targeting NR024118, si-NR024118 (CCA CCACCATCTTCCTCAATGGCAA), were designed and synthesized by Guangzhou RiboBio Co., Ltd., as were the negative control siRNAs for knockdown experiments. Transient transfections were used to transfect $10 \mathrm{nM}$ vector $(10 \mathrm{nM}$ empty vector transfection as negative control group, NC; $10 \mathrm{nM}$ small interfering empty vector transfection as negative control group, si-NC) into $10^{6} \mathrm{AC} 16$ cells. Untransfected cells were used as the control (C) cells. Lipofectamine ${ }^{\circledR} 2000$ (Invitrogen; Thermo Fisher
Scientific, Inc.) was used to perform transient transfections with plasmids or siRNAs (40 nM). The vector was first mixed with Lipofectamine ${ }^{\circledR} 2000$ (Invitrogen; Thermo Fisher Scientific, Inc.) to prepare transfection mixture. Cells were then incubated with the transfection mixture for $6 \mathrm{~h}$, followed by washing with fresh medium. Cells were cultivated in fresh medium for another $48 \mathrm{~h}$ prior to LPS treatment. The cell transfection rates were detected by reverse transcription-quantitative (RT-q) PCR.

RNA extraction. TRIzol ${ }^{\circledR}$ (Thermo Fisher Scientific, Inc.) was used to extract total RNAs from plasma and AC16 cells $\left(10^{6} \mathrm{cell} /\right.$ well) according to manufacturer's protocol. In the cases of LPS treatment, AC16 cells were cultivated in medium containing $0,150,300$ or $500 \mathrm{ng} / \mathrm{ml}$ LPS for $24 \mathrm{~h}$ before use. All RNA samples were incubated with gDNA eraser (Takara Biotechnology Co., Ltd.) at $37^{\circ} \mathrm{C}$ for $1 \mathrm{~h}$ to remove genomic DNA.

$R T-q P C R$. Precision nanoScript2 Reverse Transcription kit (Primerdesign Ltd.) was used to reverse transcribe RNA samples into cDNA and SYBR ${ }^{\circledR}$ Green Quantitative RT-qPCR kit (Sigma-Aldrich; Merck KGaA) was used to prepare all qPCR reactions all according to the manufacturers' protocols. The expression levels of NR024118 were determined with $18 \mathrm{~S}$ rRNA as endogenous control. The PCR conditions were; $95^{\circ} \mathrm{C}$ for $30 \mathrm{sec}, 95^{\circ} \mathrm{C}$ for $10 \mathrm{sec}$ and $60^{\circ} \mathrm{C}$ for $35 \mathrm{sec}$ for a total of 40 cycles. Overexpression of NR024118 in AC16 cells was confirmed by RT-qPCR. In all, three replicate qPCR reactions were included in each experiment and the $2^{-\Delta \Delta C q}$ method was used to calculate the fold changes of gene expression levels (15). Primer sequences were: 5'-AGGTTGGCTGGT GTTCCAGC-3' (forward) and 5'-CACACGCATAGAGTA GTCTC-3' (reverse) for NR024118; 5'-CTACCACATCCA AGGAAGCA-3' (forward) and 5'-TTTTTCGTCACTACC TCCCCG-3' (reverse) for human 18S rRNA.

Electrophysiological study. All whole-cell patch-clamp recordings were made from single AC16 cells in the presence of LPS, using the Axopatch 200B patch clamp amplifier (Axon Instruments; Molecular Devices, LLC) at $5 \mathrm{kHz}$ in the fast-current clamp mode at $35 \pm 1^{\circ} \mathrm{C}$.

ELISA. The levels of TNF- $\alpha$, IL-1 $\beta$ and IL-6 (presented as $\mathrm{ng} / \mathrm{ml}$ ) in plasma and the cell culture medium of AC16 cells (centrifuged at $1,000 \mathrm{x}$ g for $20 \mathrm{~min}$ at $4^{\circ} \mathrm{C}$ to remove cells,) collected at $48 \mathrm{~h}$ post-transfection were detected using human TNF- $\alpha$ (cat. no. MTA00B; Abcam), IL-1 $\beta$ (cat. no. MLB00C; Abcam) and IL-6 (cat. no. ab46042; Abcam) ELISA kits.

Cell apoptosis analysis. The effects of transfection on the apoptosis of AC16 cells were analyzed by cell apoptosis assay at $48 \mathrm{~h}$ post-transfection. AC16 cells were transferred to fresh cell culture medium supplemented with LPS at a dose of $500 \mathrm{ng} / \mathrm{ml}$. Subsequently, pre-cold phosphate-buffered saline (PBS) was used to resuspend cells, followed by staining with Annexin V-FITC/PI Apoptosis Detection kit (Beijing Solarbio Science \& Technology Co., Ltd.) for $20 \mathrm{~min}$ in dark at room temperature. Flow cytometry (FACStar PLUS; 
Table I. Baseline clinical data of three groups of participants.

\begin{tabular}{lccc}
\hline & Controls $(82)$ & Sepsis patients (82) & MD patients (35) \\
\hline Age (year) & $52.3 \pm 6.3$ years old & $52.1 \pm 6.0$ years old & $51.8 \pm 6.4$ years old \\
Sex (male/female) & $50 / 32$ & $50 / 32$ & $23 / 12$ \\
Body mass index $\left(\mathrm{kg} / \mathrm{m}^{2}\right)$ & $21.04 \pm 1.34$ & $20.99 \pm 1.48$ & $20.87 \pm 1.43$ \\
White blood cells $\left(\mathrm{x} 10^{9} / \mathrm{l}\right)$ & $6.99 \pm 1.83$ & $19.34 \pm 6.77$ & $19.98 \pm 6.98$ \\
Serum creatinine $(\mathrm{mg} / \mathrm{dl})$ & $1.09 \pm 0.31$ & $1.72 \pm 0.43$ & $1.75 \pm 0.46$ \\
C-reactive protein $(\mathrm{mg} / \mathrm{l})$ & $6.00 \pm 3.11$ & $89.23 \pm 23.77$ & $93.29 \pm 20.03$ \\
Procalcitonin $(\mathrm{ng} / \mathrm{ml})$ & $0.03 \pm 0.01$ & $9.99 \pm 3.78$ & $7.98 \pm 3.12$ \\
Albumin $(\mathrm{g} / \mathrm{l})$ & $41.56 \pm 4.22$ & $24.74 \pm 4.49$ & $25.03 \pm 4.23$ \\
APACHE II score & - & $12.77 \pm 3.45$ & $12.57 \pm 3.67$ \\
SOFA score & - & $5.5 \pm 1.49$ & $5.7 \pm 1.72$ \\
\hline
\end{tabular}

APACHE II, acute physiology and chronic health evaluation II (the theoretical maximum value of APACHE II score is 71 , which 15 or more are classified as severe, and 15 or less are classified as non-severe); SOFA, sequential organ failure assessment (SOFA score is used to evaluate the organ function of critically ill patients, which includes the evaluation of $\mathrm{PaO}_{2} / \mathrm{FiO}_{2}$ oxygenation index, platelet count, bilirubin, circulatory system function, GCS score and kidney function, with higher scores indicating a greater severity).

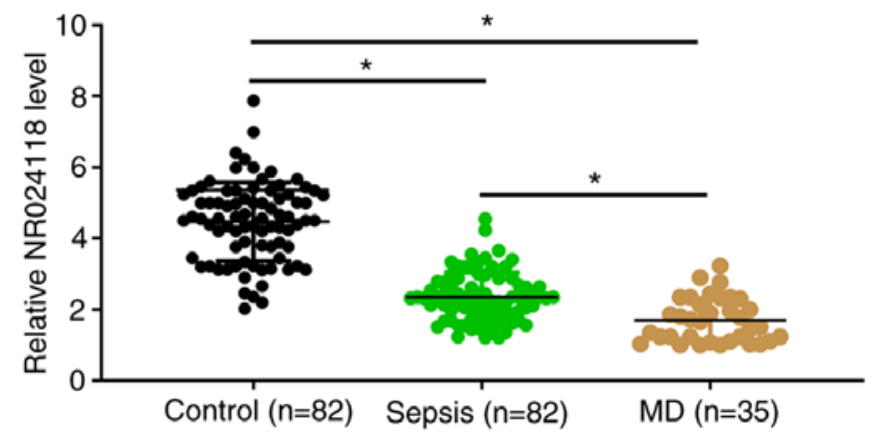

Figure 1. NR024118 is downregulated in sepsis and further downregulated in sepsis combined with MD. The expression levels of NR024118 in plasma collected from Sepsis group $(n=82)$, MD group $(n=35)$ and Control group $(n=82)$ were measured by performing reverse transcription-quantitative PCR. All PCR reactions were repeated 3 times and mean values were presented and compared. ${ }^{*} \mathrm{P}<0.05$. MD, myocardial dysfunction.

Becton, Dickinson and Company) was then used to detect the apoptotic cells. The apoptotic rate was calculated by the percentage of early + late apoptotic cells. FlowJo software (version 7.6.3; FlowJo, LLC) was used to analyze the results.

Luciferase report assays. Luciferase assay for the transcriptional activity of NF- $\kappa \mathrm{B}$ was implemented by co-transfection of a NF- $\kappa$ B TransLucent reporter vector $(\mathrm{NF}-\kappa \mathrm{B} / \mathrm{Luc}$; Panomics, Inc.; Thermo Fisher Scientific, Inc.) and a plasmid construct for $\beta$-galactosidase ( $\beta$-gal; Clontech Laboratories, Inc.) expression in AC16 cells, as a transfection control, using Lipofectamine ${ }^{\circledR}$ 2000 reagent (Invitrogen; Thermo Fisher Scientific, Inc.). Cell lysates were prepared $24 \mathrm{~h}$ after transfection, and luciferase activity was measured with the Luciferase Assay System (Promega Corporation) following the manufacturer's instructions. NF- $\kappa$ B activity were expressed after normalized to $\beta$-gal activity. BAY treatment was introduced into NR024118 overexpressing cells to inhibit the NF- $\mathrm{BB}$ pathway, with DMSO as control.
Statistical analyses. Each experiment was performed in triplicate and mean values were calculated. GraphPad Prism 6 (GraphPad Software, Inc.) software was used to perform all statistical analyses. One-way analysis of variance (ANOVA) combined with Tukey's post hoc test was used to compare differences among multiple groups. Correlations were analyzed by Pearson's correlation coefficient. $\mathrm{P}<0.05$ was considered to indicate a statistically significant difference.

\section{Results}

NR024118 is downregulated in sepsis and further downregulated in sepsis combined with MD. The expression levels of NR024118 in plasma collected from Sepsis group $(n=82)$, MD group $(n=35)$ and the Control group $(n=82)$ were measured by performing RT-qPCR. Compared with the Control group, the expression levels of NR024118 were significantly lower in Sepsis and MD groups (Fig. 1; $\mathrm{P}<0.05$ ). In addition, the expression levels of NR024118 were also significantly lower in MD group than that in Sepsis group (Fig. 1; P<0.05).

IL-6 is upregulated in sepsis and further upregulated in sepsis combined with MD. The expression levels of IL-6 in plasma collected from Sepsis group $(n=82)$, MD group $(n=35)$ and the Control group $(n=82)$ were measured by ELISA. Compared with the Control group, the expression levels of IL- 6 were significantly increased in Sepsis and MD groups (Fig. 2; $\mathrm{P}<0.05)$. In addition, the expression levels of IL-6 were also significantly higher in MD group than that in Sepsis group (Fig. 2; P<0.05).

NR024118 and IL-6 are inversely correlated in sepsis with MD. The correlation between the expression levels of NR024118 and IL- 6 across plasma samples from MD group $(n=35)$ were analyzed by Pearson's correlation coefficient. It was observed that NR024118 and IL-6 were inversely and significantly 


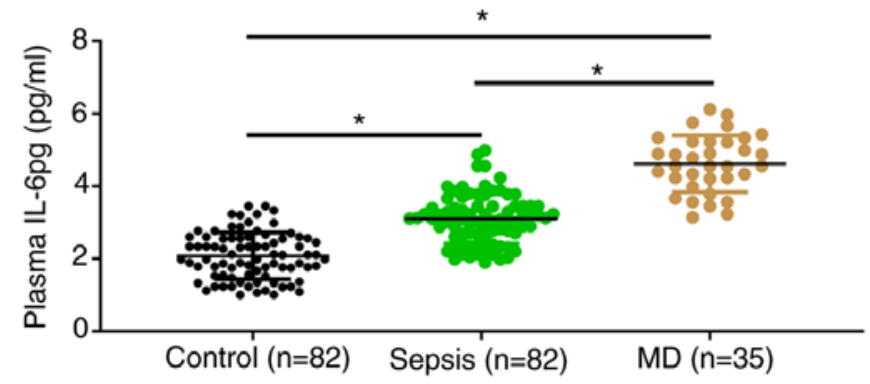

Figure 2. IL-6 is upregulated in sepsis and further upregulated in sepsis combined with MD. The expression levels of IL-6 in plasma collected from Sepsis group $(n=82)$, MD group $(n=35)$ and Control group $(n=82)$ were measured by ELISA. All measurements were repeated 3 times and mean values were presented and compared. ${ }^{\mathrm{P}} \mathrm{P}<0.05$. MD, myocardial dysfunction.

correlated (Fig. 3). The close correlation indicated possible interaction.

LPS can induce MD. To determine the effect of LPS on myocardial cells, AC-16 cells were treated with different concentrations $(0,150,300$ and $500 \mathrm{ng} / \mathrm{ml})$ of LPS. Flow cytometry results demonstrated that the apoptosis ability of AC-16 cells was enhanced with the increase of LPS concentration (Fig. 4A). Simultaneously, the APD in the LPS group were significantly shorter compared with that in the control group (Fig. 4B). In addition, to determine whether myocardial cells had an inflammatory response, the contents of inflammatory cytokines were measured. ELISA demonstrated that the amount of TNF- $\alpha$, IL- $1 \beta$ and IL- 6 were enhanced with the increase of LPS concentration, indicating the increased inflammatory response of AC-16 cells (Fig. 4B-E). These data confirmed that LPS could induce the expression of inflammatory factors and shorten APD to construct sepsis-induced MD.

Overexpression of NR024118 reduces the secretion of IL-6 from cardiomyocytes and ameliorated LPS-induced myocardial APD duration and cell injury. AC16 cells were cultivated in medium containing LPS $(0,150,300$ or $500 \mathrm{ng} / \mathrm{ml})$ for $24 \mathrm{~h}$, followed by measurement of the expression levels of NR024118 by RT-qPCR. It was observed that LPS treatment decreased the expression levels of NR024118 in AC16 cells in a dose-dependent manner (Fig. 5A; $\mathrm{P}<0.05$ ). Subsequently, AC16 cells were transfected with NR024118 expression vector. Overexpression of NR024118 in AC16 cells were confirmed by RT-qPCR. Compared with the $\mathrm{C}$ (untransfected cells) and $\mathrm{NC}$ (empty vector transfection) groups, the expression levels of NR024118 were significantly increased at $48 \mathrm{~h}$ post-transfection (Fig. 5B; $\mathrm{P}<0.05)$. In addition, Compared with the $\mathrm{C}$ and si-NC (small interfering empty vector transfection) group, the expression levels of NR024118 were significantly decreased by si-NR024118. In addition, $\mathrm{APD}_{90}$ duration following the overexpression of NR024118 in AC16 cells were longer (Fig. 5C; $\mathrm{P}<0.05)$. ELISA was performed to analyze the secretion of IL-16 from AC16 cells in each transfection group. Compared with $\mathrm{C}$ and $\mathrm{NC}$ groups, IL-6 secretion was significantly reduced in AC16 cells with the overexpression of NR024118, while silencing of NR024118 demonstrated the opposite

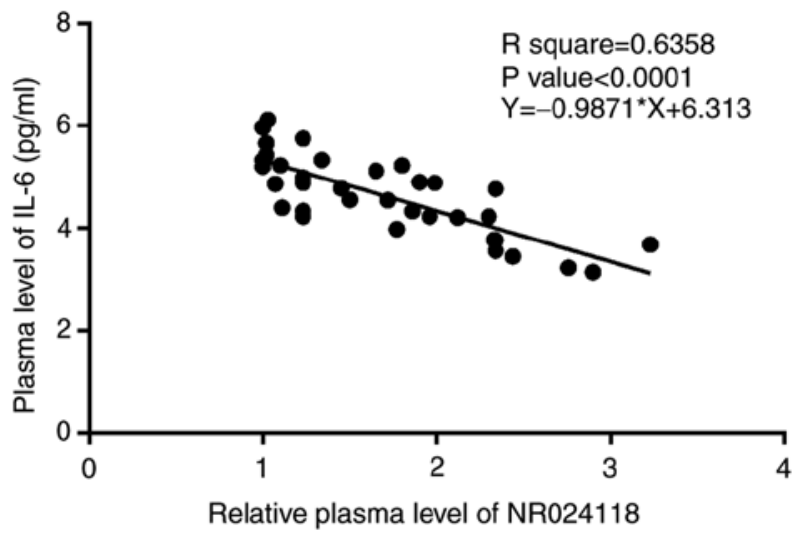

Figure 3. NR024118 and IL-6 are inversely correlated with in sepsis with MD. Pearson's correlation coefficient was used to analyze the correlation between plasma levels of NR024118 and IL-6 across plasma samples from MD group ( $n=35)$. MD, myocardial dysfunction.

results (Fig. 5D; $\mathrm{P}<0.05)$. Cell apoptosis assay was performed to assess the effects of NR024118 on LPS-induced apoptosis of AC16 cells. Compared with $\mathrm{C}$ and $\mathrm{NC}$ groups, overexpression of NR024118 significantly decreased the apoptosis of AC16 cells, while silencing of NR024118 had the opposite effect (Fig. 5E; P<0.05).

NR024118 regulates LPS-induced MD through the NF- $\kappa B$ signaling pathway. The $\mathrm{NF}-\kappa \mathrm{B}$ signaling pathway is a classic signaling pathway associated with inflammatory response. To determine whether LPS-induced cellular inflammation response could be achieved by regulating the $\mathrm{NF}-\kappa \mathrm{B}$ signaling pathway, the effect of NR02411 on the NF- $\kappa \mathrm{B}$ signaling pathway in LPS-treated AC16 cells was evaluated. Western blot analysis demonstrated that p65 phosphorylation (Fig. 6A) and NF- $\kappa \mathrm{B}$ activation (Fig. $6 \mathrm{~B}$ ) were inhibited by overexpression of NR02411 while promoted by inhibition of NR02411. In addition, IL- 6 production was also elevated in cardiomyocytes with the silencing of NR02411, while this effect can be counteracted by further treatment with BAY (Fig. 6C). These results demonstrated that NR02411 could negatively regulate IL-6 production by inhibiting p65 phosphorylation and the $\mathrm{NF}-\kappa \mathrm{B}$ signaling pathway.

\section{Discussion}

The involvement of NR024118 in sepsis and sepsis-induced MD was investigated in the present study. It was found that NR024118 was downregulated in sepsis and further downregulated in sepsis with MD. NR024118 may suppress the secretion of IL-16 and cell apoptosis to improve sepsis.

The functions of NR024118 have been mainly investigated in inflammatory responses $(12,16)$. It has been reported that NR024118 can interact with the $\mathrm{NF}-\kappa \mathrm{B} / \mathrm{Nrf} 2$ signaling to suppress LPS-induced cell apoptosis and inflammatory responses in chondrocytes (12). In a rheumatoid arthritis rat model, the expression of NR024118 in synovial fibroblasts can be induced by Shikonin to suppress inflammatory responses (16). These two studies have characterized NR024118 as an inhibitor of inflammation. Sepsis is essentially a type of inflammatory disease (1). Consistently, the 

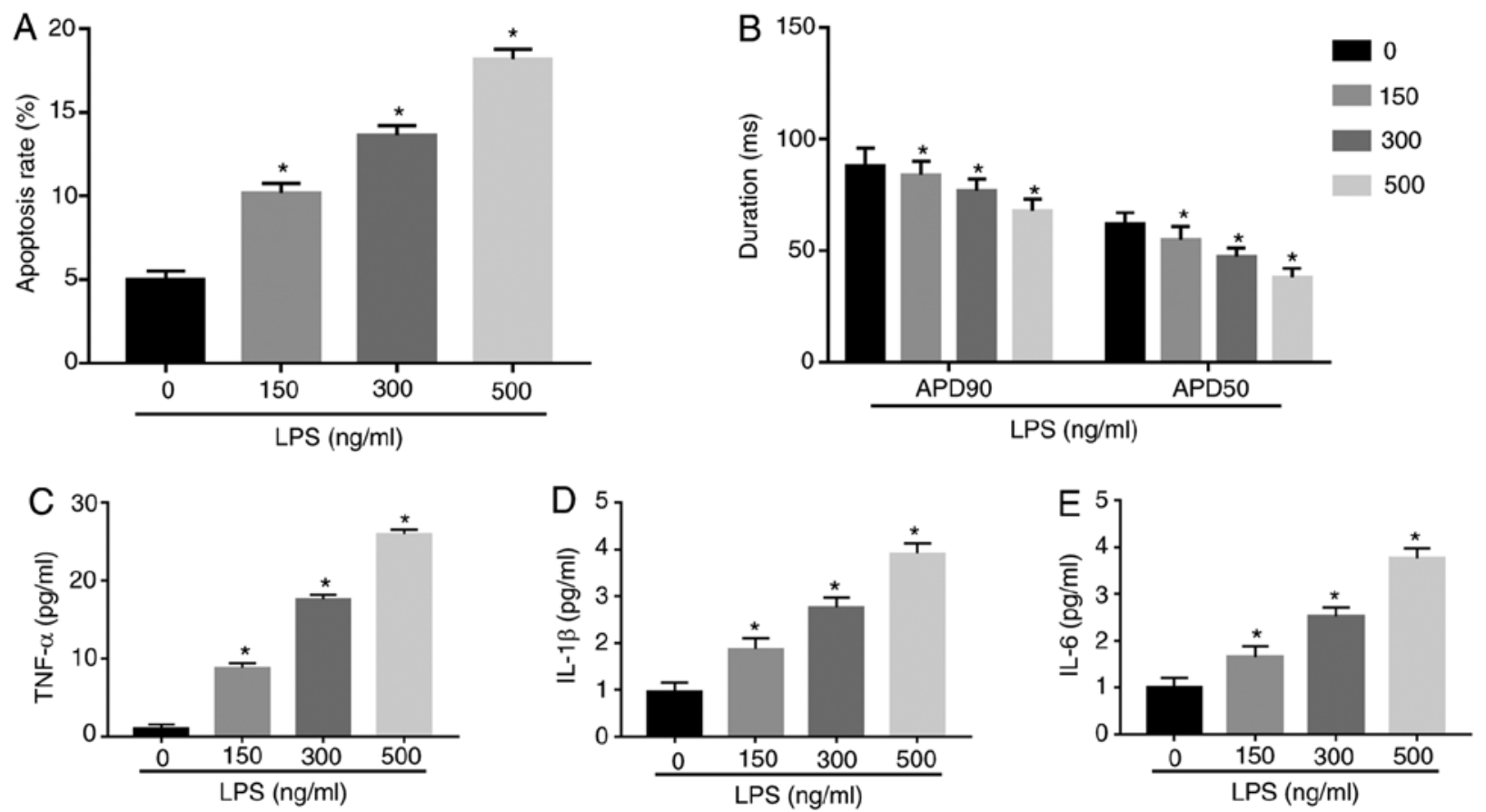

Figure 4. LPS can induce MD. AC16 cells were treated with different concentrations (0, 150, 300 and $500 \mathrm{ng} / \mathrm{ml})$ of LPS. (A) Apoptosis rate and (B) APD of AC16 cells were measured by flow cytometry and the patch-clamp. Concentration of (C) TNF- $\alpha$, (D) IL-1 $\beta$ and (E) IL- 6 were determined by ELISA. All experiments were performed in triplicate and mean values were presented and compared. " $\mathrm{P}<0.05$. LPS, lipopolysaccharide; MD, myocardial dysfunction; APD, action potential duration.
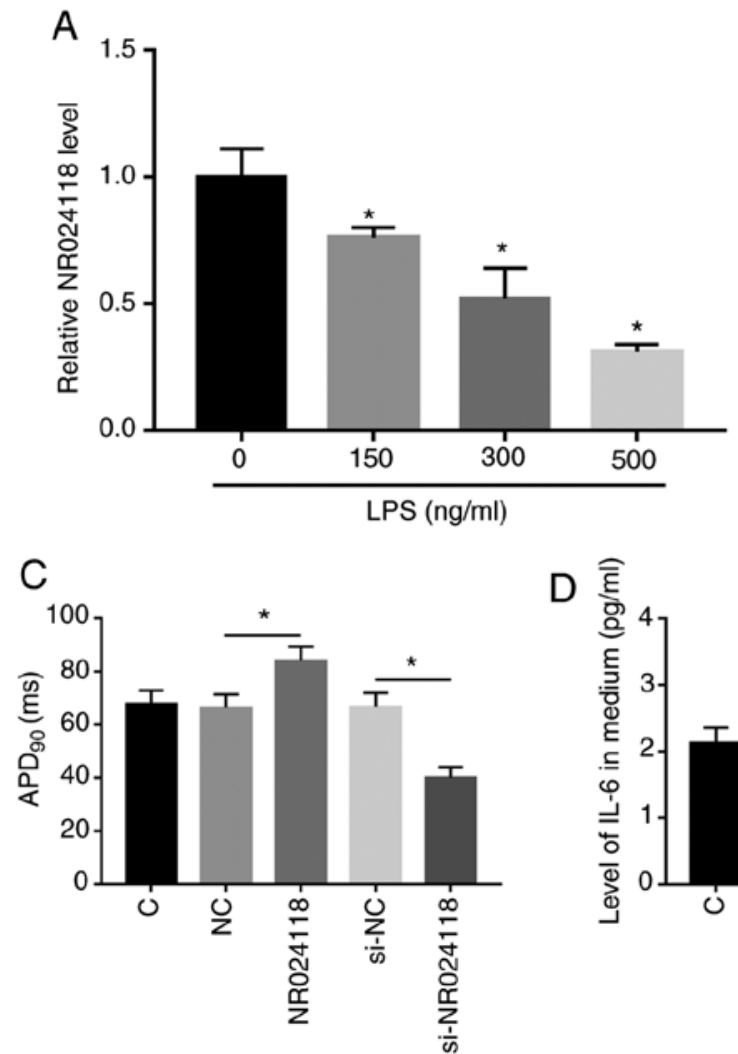

$\mathrm{D} \widehat{\widehat{\varepsilon}}$
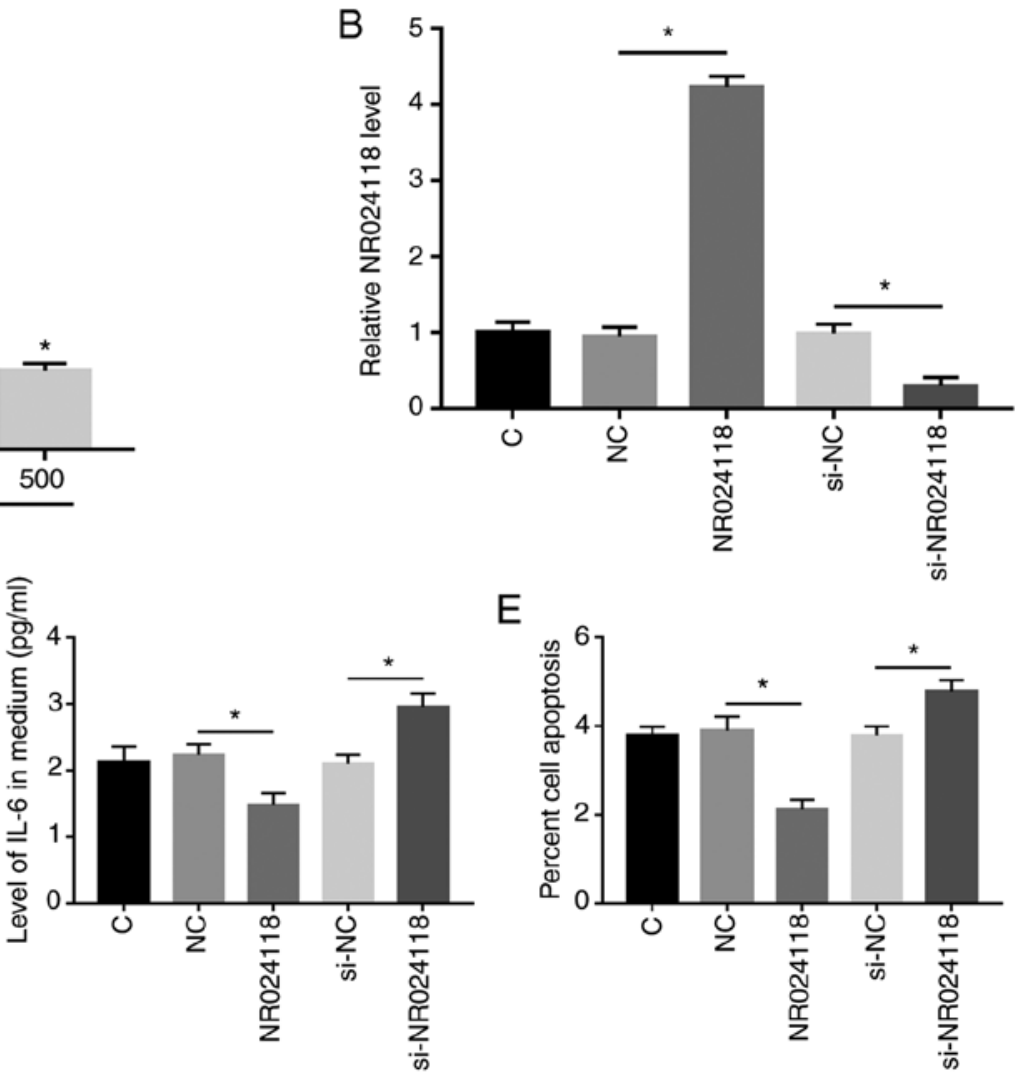

E

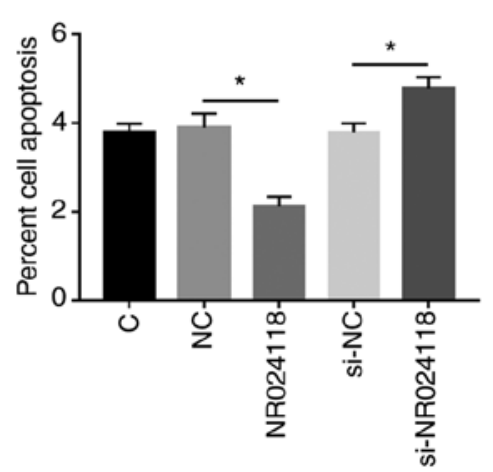

Figure 5. Overexpression of NR024118 reduces the secretion of IL-6 from cardiomyocytes and ameliorates LPS-induced myocardial APD duration and cell injury. (A) AC16 cells were cultivated in medium containing 0,150,300 or $500 \mathrm{ng} / \mathrm{ml} \mathrm{LPS} \mathrm{for} 24 \mathrm{~h}$, followed by the measurement of expression levels of NR024118 by reverse transcription-quantitative (RT-q) PCR. (B) Overexpression of NR024118 in AC16 cells was confirmed $48 \mathrm{~h}$ post-transfection by performing qPCR. (C) $\mathrm{APD}_{90}$ of AC16 cells were measured by flow cytometry and patch-clamp. (D) ELISA was performed to analyze the secretion of IL-16 from AC16 cells in each transfection group. (E) Cell apoptosis assay was performed to analyze the effects of NR024118 on LPS-induced apoptosis of AC16 cells. All experiments were performed in triplicate and mean values were presented and compared. "P $<0.05$. APD, action potential duration; LPS, lipopolysaccharide; APD, action potential duration; C, control; NC, negative control; si, short interfering. 
A
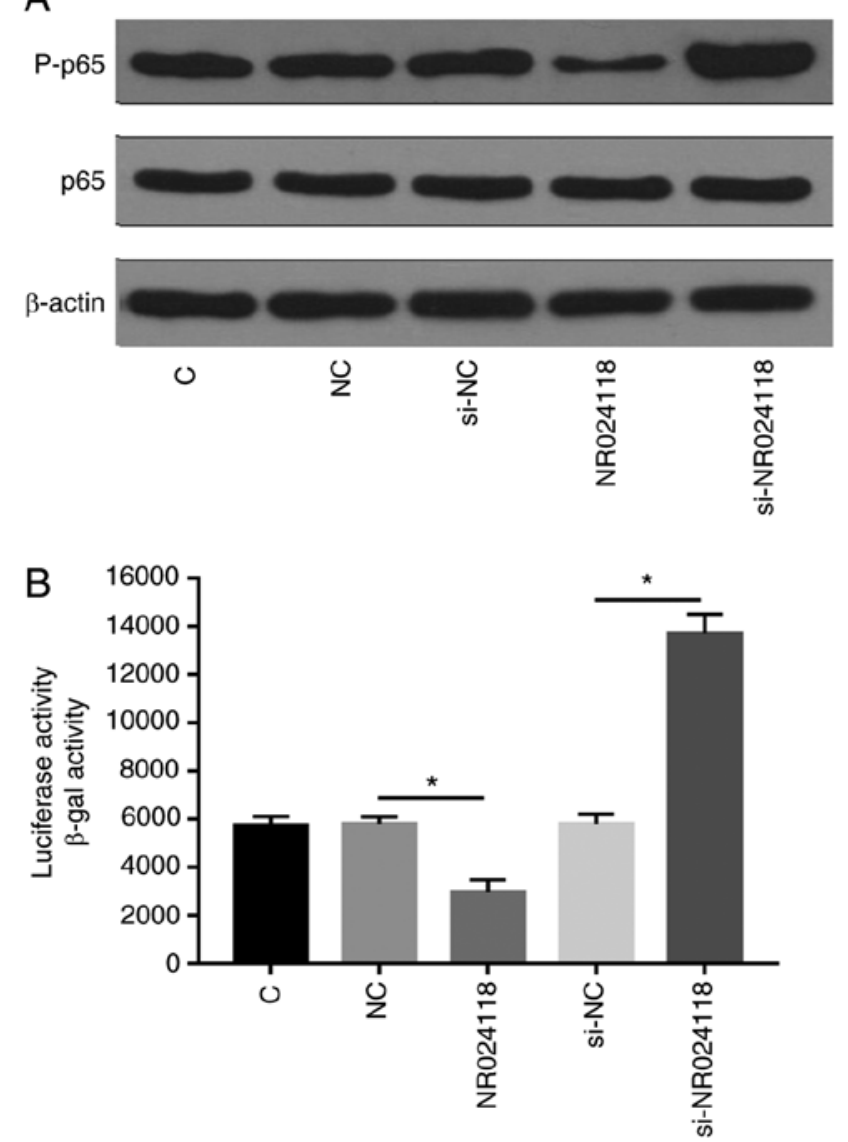
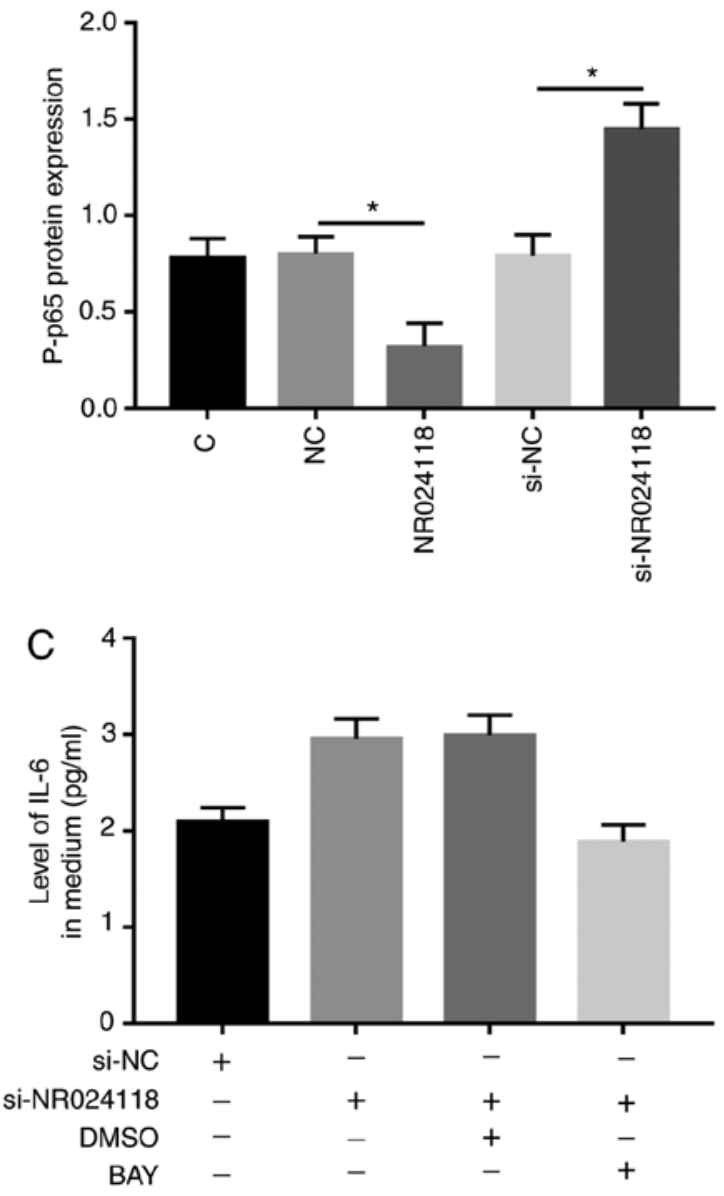

Figure 6. NR024118 regulates LPS-induced MD through the NF-кB signaling pathway. AC16 cells were transfected with NR024118 or si-NR024118 for NR024118 inhibition or overexpression separately. (A) The expression of p-p65 and the ratio of p-p65/p65 was detected by western blotting. (B) NF- $\kappa$ B activity were detected by luciferase report assays. (C) BAY treatment was introduced into NR024118 silencing cells to inhibit NF- $\kappa$ B pathways for the analysis of IL- 6 production. All experiments were performed in triplicate and mean values were presented and compared. * $\mathrm{P}<0.05$. LPS, lipopolysaccharide; MD, myocardial dysfunction; p-, phosphorylated; C, control; NC, negative control; si, short interfering.

present study also observed the downregulation of NR024118 in sepsis, indicating the involvement of NR024118 in sepsis. A previous study reported that in cardiac fibrosis angiotensin II induces the downregulation of NR024118 to promote disease progression (17), indicating the involvement of NR024118 in heart diseases. In the present study further downregulation of NR024118 was observed in patients with sepsis combined with MD. In addition, overexpression of NR024118 decreased heart cell apoptosis. Therefore, NR024118 may have protective effects on sepsis induced MD.

IL-6 is a major contributor to inflammatory responses in patients with sepsis (14). The present study also observed further upregulation of IL-6 in patients with sepsis with MD compared with patients with sepsis without MD. Therefore, the further upregulation of IL-6 may promote the development of MD. The present study observed an inverse correlation between NR024118 and IL-6 across plasma samples from patients with sepsis complicated with MD. LPS is often used to induce cellular sepsis models in vitro due to its ability to bind to Toll-like receptor 4 and activate inflammatory response (18). In the LPS-treated myocytes, APD was significantly shortened under sepsis (19). The effect of LPS on myocardial cells was evaluated and it was found that LPS could promote apoptosis as well as the amounts of TNF- $\alpha$, IL-1 $\beta$ and
IL-6, indicating the increased inflammatory response of AC16 cells. Simultaneously, the APD in the LPS group was shorted significantly compared with that in the control group.

In summary, the results of the present study demonstrated that NR024118 was significantly inhibited in LPS-induced AC16 cells and overexpression of NR024118 reduced IL-6 secretion and increased APD, while silencing of NR024118 had the opposite effect. It has been well established that IL-6 can promote inflammatory responses in patients with sepsis to aggregate disease conditions (14). Therefore, NR024118 suppresses the secretion of IL-6 to relieve sepsis and prevent sepsis induced MD. In addition, p65 phosphorylation and $\mathrm{NF}-\kappa \mathrm{B}$ activation were inhibited by overexpression of NR02411 and promoted by inhibition of NR02411. This suggested that NR02411 alleviated the inflammatory response of LPS-induced myocardial cells mainly by inhibiting the $\mathrm{NF}-\kappa \mathrm{B}$ signaling pathway. Therefore, it was concluded that NR024118 was suppressed in sepsis and inhibited LPS-induced apoptosis of cardiomyocytes. However, the present study only included in vitro cell experiments, which may not fully reflect the disease conditions in the heart of patients with sepsis. Future studies may explore the expression of NR02411 in sepsis-induced myocardial tissues and analyze the function of NR02411 in vivo through animal models. 
In conclusion, NR024118 was downregulated in sepsis and sepsis-induced MD. NR024118 may interact with IL-6 and regulate heart cell apoptosis to participate in sepsis induced MD.

\section{Acknowledgements}

Not applicable.

\section{Funding}

No funding was received.

\section{Availability of data and materials}

The analyzed data sets generated during the study are available from the corresponding author on reasonable request.

\section{Authors' contributions}

GQ and LW were responsible for study design, writing the manuscript, literature searches, performing experiments and data analysis. FJ and JL were responsible for literature searches, performing experiments and data analysis. BZ, DP and XL were responsible for data acquisition and statistical analysis. GQ and LW confirmed the authenticity of all the raw data. All authors read and approved the final manuscript.

\section{Ethics approval and consent to participate}

The present study was approved by the Ethics Committee Guangxi Zhuang Autonomous National Hospital. The present study was performed in accordance with the World Medical Association Declaration of Helsinki. All patients provided written informed consent prior to their inclusion within the present study.

\section{Patient consent for publication}

Not applicable.

\section{Competing interests}

The authors declare that they have no competing interests.

\section{References}

1. Hotchkiss RS, Moldawer LL, Opal SM, Reinhart K, Turnbull IR and Vincent JL: Sepsis and septic shock. Nat Rev Dis Primers 2: 16045,2016

2. Moore JX, Donnelly JP, Griffin R, Howard G, Safford MM and Wang HE: Defining sepsis mortality clusters in the United States. Crit Care Med 44: 1380-1387, 2016.

3. Resch B, Gusenleitner W and Müller WD: Procalcitonin and interleukin-6 in the diagnosis of early-onset sepsis of the neonate. Acta Paediatr 92: 243-245, 2003.
4. Krishnagopalan S, Kumar A, Parrillo JE and Kumar A: Myocardial dysfunction in the patient with sepsis. Curr Opin Crit Care 8: 376-388, 2020.

5. Kakihana Y, Ito T, Nakahara M, Yamaguchi K and Yasuda T: Sepsis-induced myocardial dysfunction: Pathophysiology and management. J Intensive Care 4: 22, 2016.

6. Ibrahim MH, Azab AA, Kamal NM, Salama MA, Ebrahim SA, Shahin AM, El-Sadek AE, Abdulghany WE, Sherief LM and Abdallah EA: Early detection of myocardial dysfunction in poorly treated pediatric thalassemia children and adolescents: Two Saudi centers experience. Ann Med Surg (Lond) 9: 6-11, 2016.

7. Englert JA, Bobba C and Baron RM: Integrating molecular pathogenesis and clinical translation in sepsis-induced acute respiratory distress syndrome. JCI Insight 4: e124061, 2019. (Online ahead of print).

8. Rossaint J and Zarbock A: Pathogenesis of multiple organ failure in sepsis. Crit Rev Immunol 35: 277-291, 2015.

9. Zhang TN, Li D, Xia J, Wu QJ, Wen R, Yang N and Liu CF: Non-coding RNA: A potential biomarker and therapeutic target for sepsis. Oncotarget 8: 91765-91778, 2017.

10. Fang Y, Hu J, Wang Z, Zong H, Zhang L, Zhang R and Sun L: LncRNA H19 functions as an Aquaporin 1 competitive endogenous RNA to regulate microRNA-874 expression in LPS sepsis. Biomed Pharmacother 105: 1183-1191, 2018.

11. Shen J, Zhang J, Jiang X, Wang H and Pan G: LncRNA HOX transcript antisense RNA accelerated kidney injury induced by urine-derived sepsis through the miR-22/high mobility group box 1 pathway. Life Sci 210: 185-191, 2018.

12. Mei X, Tong J, Zhu W and Zhu Y: lncRNA-NR024118 overexpression reverses LPS-induced inflammatory injury and apoptosis via NF- $\mathrm{B} / \mathrm{Nrf2}$ signaling in ATDC5 chondrocytes. Mol Med Rep 20: 3867-3873, 2019.

13. He G, Zhang X, Chen Y, Chen J, Li L and Xie Y: Isoalantolactone inhibits LPS-induced inflammation via NF- $\kappa$ B inactivation in peritoneal macrophages and improves survival in sepsis. Biomed Pharmacother 90: 598-607, 2017.

14. Hou T, Huang D, Zeng R, Ye Z and Zhang Y: Accuracy of serum interleukin (IL)-6 in sepsis diagnosis: A systematic review and meta-analysis. Int J Clin Exp Med 8: 15238-15245, 2015.

15. Livak KJ and Schmittgen TD: Analysis of relative gene expression data using real-time quantitative PCR and the 2(-Delta Delta C(T)) method. Methods 25: 402-408, 2001.

16. Yang KY and Chen DL: Shikonin inhibits inflammatory response in rheumatoid arthritis synovial fibroblasts via lncRNA-NR024118. Evid Based Complement Alternat Med 2015: 631737, 2015.

17. Jiang $X$, Zhang $F$ and Ning Q: Losartan reverses the down-expression of long noncoding RNA-NR024118 and Cdkn1c induced by angiotensin II in adult rat cardiac fibroblasts. Pathol Biol (Paris) 63: 122-125, 2015.

18. Wei JL, Wu CJ, Chen JJ, Shang FT, Guo SG, Zhang XC and Liu H: LncRNA NEAT1 promotes the progression of sepsis-induced myocardial cell injury by sponging miR-144-3p. Eur Rev Med Pharmacol Sci 24: 851-861, 2020.

19. Tai BY, Wen ZH, Cheng PY, Yang HY, Duh CY, Chen PN and Hsu CH: Lemnalol modulates the electrophysiological characteristics and calcium homeostasis of atrial myocytes. Mar Drugs 17: 619,2019

This work is licensed under a Creative Commons Attribution 4.0 International (CC BY-NC 4.0) License 